\title{
The problem of SEO promotion for the organization's web representation
}

\author{
Danil Gek ${ }^{1}$, Vladislav Kukartsev ${ }^{1,2}$, Vadim Tynchenko ${ }^{1,2, *}$, Aleksandr Bondarev $^{1}$, Maria Pokushko $^{2}$, \\ and Natalya Dalisova ${ }^{2}$ \\ ${ }^{1}$ Reshetnev Siberian State University of Science and Technology, 31 Krasnoyarsky Rabochy av., Krasnoyarsk, Russia \\ ${ }^{2}$ Siberian Federal University, 79 Svobodny pr., Krasnoyarsk, Russia
}

\begin{abstract}
The article describes the problem of search engine optimization of a new web-site of any organization and the approaches to make better results in different search engines. The new site is difficult to bring to the top search engine rankings. This article describes the study of the new web-site SEOpromotion. The authors took the site of the organization for the sushi and rolls delivery in Krasnoyarsk city, Russian Federation, which was created and uploaded to the hosting about a year ago. At the first stage, the technical optimization was completed: Robots.txt and Sitemap.xml files were configured, 404 page was set up and validation check was carried out. At the second stage, the content was worked out and a semantic core was formed. With the help of the Yandex service Wordstat.Yandex, key phrases were selected, which was used to advance the site. After that, a snippet was created which makes the link to the site more attractive to users. As a result of this work, the site has become 2 times more visible to potential customers of the company. Thus, properly implemented SEO optimization allows you to attract a significantly larger number of customers in the company.
\end{abstract}

\section{Introduction}

Despite universal appreciation and popularity, the Internet continues to expand its borders. According to WeAreSocial, an international social media research agency, in their Q4 Global DigitalStatshot report for the fourth quarter of 2018, more and more people in the developing world are connecting to the Internet [1]. Millions of people across Africa and South Asia have connected to the network for the first time in the last three months, with almost all of these new users accessing the Internet exclusively through mobile devices [2]. In October 2018, there were almost 4.2 billion Internet users in the world. Since September 2017, 284 million new users have appeared on the network. In Russia, the Internet audience has also increased by $4 \%$. They reached 90 million people as of September 2017 - February 2018, which is about $73 \%$ of the country's population over 12 years old.

These data suggest that the Internet is an integral part of a person's daily life [3]. People find answers to various questions (personal, workers), communicate in social networks, and satisfy their consumer needs with the help of the World Wide Web. In the 21st century, a person does not wander around the city in search of the store and goods he needs. Before buying something, he will go online, write a query in the search box and start wandering through the pages of the Internet in search of an answer to his question [4 - 6].
The Internet is a broad community of millions of users, and it is also named as one of the most important means of communication today. This wider community is also known as an important customer potential for anyone thinking about business activities based on the Internet environment [7]. The Internet has become a new channel of the business sphere in the modern world, in which it is impossible to achieve such a large volume using traditional sales channels. Work methods have changed with the advent of the Internet and created some changes in other areas [8 - 10]. Companies are in dire need of internet marketing. Not a single company, even the smallest, can manage today without its own website. Entrepreneurs and businessmen are aware of the need to present themselves and their services on virtual pages. Large chains and stores create copies of themselves online, offering their entire range of products. After all, it is beneficial because [11 - 14]:

- On the Internet, there is an opportunity to reach a huge audience of potential buyers, a chance to quickly make themselves known.

- To make business through Internet is cheaper than rent office or retail space where large areas, furniture, and staff are required. The web site cost less, with a constant influx of buyers.

- Making purchases over the Internet is easier and more economical in terms of time. In addition, evaluate the product and make comparison is often performed by users before the purchase itself, and they are made, as it is not difficult to guess, on the Internet.

\footnotetext{
"Corresponding author: vadimond@mail.ru
} 
- Ability to tell about yourself, presenting the most important information in a stylish way. Create an image.

At the first time of website launching, it is likely that a couple of users will enter it, either by accident or as a result of a targeted search for this resource. This happens because on the Internet there are countless various sites, both offering any products, and just filled with a variety of information [15 - 17]. While the Internet includes hundreds of thousands of web-pages, it is best to use search engines to find the right data or information. According to a study conducted in February 2016, 81\% of Internet users visit the search web pages. In 2019, Google.com became the most popular No. 1 website on the world market and in the United States. Those companies that have realized how wide the possibilities of the Internet are, the volume of potential buyers, have long occupied the best places. These places that any web user met are on the pages of search engines, and the best ones are always displayed on the first page on request and are called Top 10. Not many, having received a selection of the necessary resources on the first page, start flipping through the following, and even more so, rarely anyone reaches at least the fifth page, well, the place of only the created site can be much further [18]. Because of this, it is important that the web page get to the top of the search results list. To achieve this goal, website developers should use search engine optimization [19]. You can move a web page to the first page of a search engine using only optimization rules without paid online advertising. Therefore, it is of great importance to promote the site, its conclusion in the ranking using search engine optimization.

Search engine optimization (SEO) is defined as a set of measures for external and internal website optimization to increase its place in the final lists of search engines for certain requests of users. It is in order to increase the flow of network traffic and potential buyers and profit from incoming users. [20-21]

\section{Problem statement}

The new site is difficult to bring to the top search engine rankings. The leading places are occupied by sites that have long been familiar to robots, as well as sites that paid for contextual advertising. While SEO takes time, search marketing (SEM) allows you to save it and automatically appear on a web page in front of a target audience actively searching for products and services.

Advertising in search engines (also known as search advertising, online advertising or search advertising on the Internet) allows you to directly display paid ads in search results in various search engines such as Google, Yandex, Bing and Yahoo. Advertisers using search advertising show their ads to users who are actively searching for specific keywords, and they pay for each click on their ad. The issuance of search engines for any competitive request is accompanied by a display of a group of advertisements - they are located above and below the main issue of the results of search ranking sites.
In different search engines for various requests shows a different number of advertising sites. On figures 1 and 2 three advertisements are shown from Yandex and Google search engines (Figures 1 and 2).

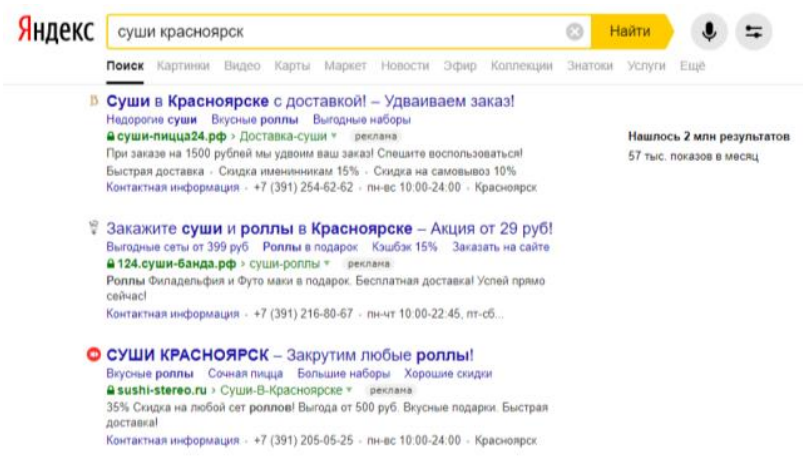

Fig. 1. Advertisements in Yandex (web-site language is Russian)

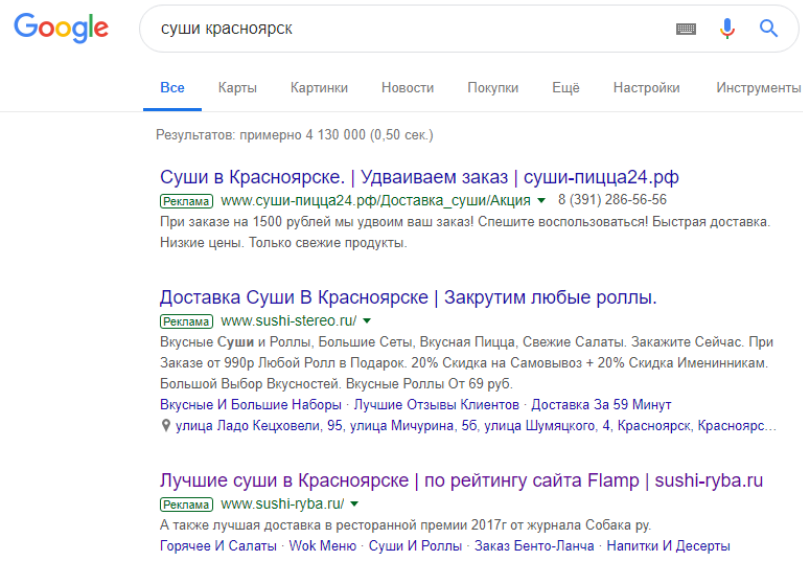

Fig. 2. Advertisements in Google (web-site language is Russian)

A small number of positions are left for organic delivery; therefore, it is a difficult task to get place in the top for free. To do this, it is needed to maximize the quality of the site, to use all the available tools of SEOpromotion and to wait.

\section{Description of the experiment}

This article describes the study of web site SEOpromotion. The authors took the site of the organization for the sushi and roll delivery «Sushi Boom». This site was created and uploaded to the hosting about a year ago. Due to the lack of any SEO-settings, it was not indexed by search robots, so the website is unknown for them. Such a website can increase the number of sales by accessing complete information about products and consumer services on the Internet. In general, the structure of the site directory is represented by three pages:

- Home - information about the company,

- Menu - product catalogue,

- Contacts - contact details (address, phone number, hours of operation). 
The main page contains the information about the company:

- Name,

- Description of activities,

- Contacts.

On the Menu page, visitors can familiarize themselves with the presented assortment of rolls (name, composition, weight, quantity and price) and drinks (name, volume and price), phone number to order.

In addition, each page has a so-called «header». This is the upper part of the site, usually consisting of the company name, logo, navigation bar, basic contact information. The header is one of the main design elements of the web platform, its attractiveness on the web and the convenience of using the site. It is also important for usability to provide visitors with key information about the resource. The main elements of the header should give answers to users' questions: what is the company, what does it offer, how to contact it, what are the promotions, guarantees, etc.

Site footer (basement) is a structural element located at the bottom of the page. It is used as a feedback tool: it contains a button for the appearance of a modal window with a form for reviews. The feedback form is the most acceptable way to communicate between the owner of the resource and the user.

Yandex represents a large number of tools for web developers, analysts, SEO-specialists and companies in general. These tools have wide functionalities and are more informative than similar ones. The site sushiboom24.ru has been added to the «Yandex.Webmaster» service. It shows site statistics, indexing errors, data on external and internal links, allows you to check the correctness of technical files on the site, manage the site view in the search results. An organization card was also filled in the «Yandex.Spravochnik» service. This service contains information about organizations and shows to users on Yandex search services. The filled profile will help the organization to appear in the search results of the Maps more often according to different filters. Yandex will bring a variety of contact information to users. Filling an organization's profile is also completely beneficial because the Yandex ranking algorithm automatically raises the most informative company cards in the results. According to Yandex statistics, completed cards are shown $20 \%$ more often. Based on the above-described advantages of Yandex services, it was decided to follow the website promotion in this search engine.

\section{Technical optimization}

At the first stage of the project, the technical side of the website has been worked out. Important technical files Robots.txt and Sitemap.xml were configured. The Robots.txt file contains direct instructions to search engines about the pages to add to the search database. Search robots were prohibited from indexing the Fonts directory containing the site fonts and the PHP feedback form processing file. The main mirror of the website without "www" (https://sushoboom24.ru) was created.
This setting is necessary for the search engines to index the site with "www" and without "www" as a whole. The site mirror is an exact or almost exact copy of the site, accessible at different addresses. The robot will not be confused when finding mirrors of the site and will understand that the main mirror is specified in the robots.txt file. Also, the path to the Sitemap.xml file was specified in robots.txt file. A sitemap is a file with links to the pages of the site that tells search engines about the current structure of the site.

The site has set up a 404 page. When accessing a non-existent page, the server must inform the "404 NotFound" error to browser, and the browser will inform the user by displaying a special page, the so-called "stub", indicating the user about errors in the entered address (Figure 3).

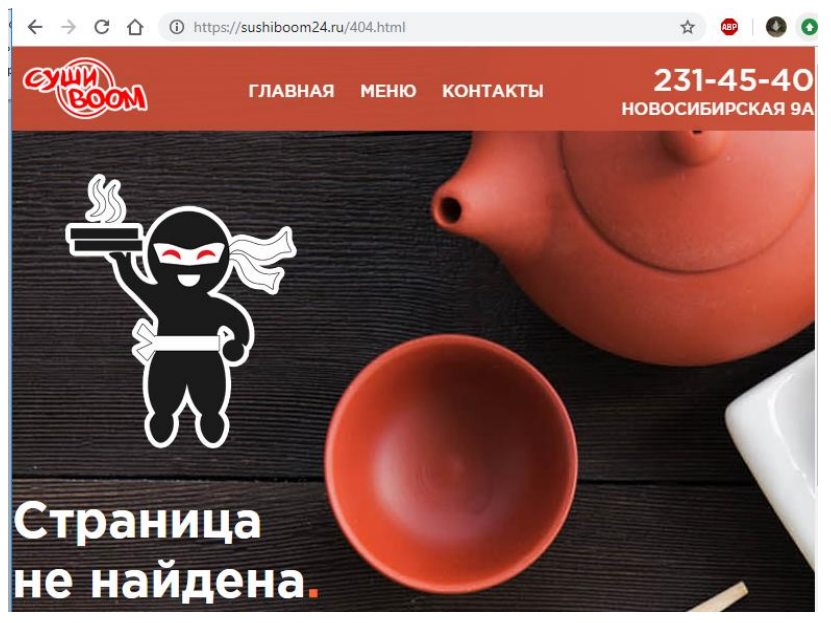

Fig. 3. Page 404 (web-site language is Russian)

Next, a validation check was carried out. Check mark-up is an important step towards the technical quality of web pages. Code validity is its compliance with existing HTML formatting standards: no syntax errors, tag nesting and much more. Valid code fully meets the requirements of the World Wide Web Consortium W3C, which develops common principles and standards of the Internet. Check W3C allows the site to get the best ranking in search engines. Search engines check the HTML code of the site when searching. If they find invalid HTML code, that is, code that does not follow official rules, the site can be removed from their indexes. If there is an error in the code of the web page, the robots will stop searching the entire content of the website. To avoid this, all found errors in the code were corrected (Figure 4).

In parallel with checking the validity of the site, a check was carried out for the absence of duplicate pages, content, meta tags. Search robots have a very negative attitude to duplicate texts, consider sites with duplicate non-unique and unhealthy for users and, therefore, reduce their place in the issue.

One of the important steps of SEO site optimization is image optimization. A comprehensive SEO strategy includes a plan for creating images that resonate with your audience, while remaining relevant to your content. Google itself recognizes the importance of images and 
shares helpful tips on image optimization in its image publishing guidelines.

Nu Html Checker
This tool is an ongoing experiment in better HTML checking, and its behavior remains
subject to change
Showing results for https://sushiboom24.ru/
Checker Input
Show source outline image report Options...
Check by address
https://sushiboom24.ru/
Check

Document checking completed. No errors or warnings to show.

Used the HTML parser.

Total execution time 808 milliseconds.

About this checker $\cdot$ Report an issue $\cdot$ Version: 19.3.30

Fig. 4. Checking the validity of the code

All images on the site are of high quality, consistent with the content and theme of the site, have high resolution. Using the service TinyPNG, all images on the site were optimized. Reducing the overall size of images has led to an increase in the speed of pages loading. Pages that take a lot of time often suffer from a high failure rate due to the quick departure of active visitors looking for an immediate solution. The alt attribute was also checked for all images on the pages. It should not be empty. Alternative text makes it possible to increase content in terms of search engine optimization.

\section{Semantics}

After setting up the technical side of the site, the next stage of optimization was preceded: study of its semantics. At the second stage, the content was worked out and a semantic core was formed. The semantic core is an ordered set of words, their morphological forms and phrases that most accurately characterize the type of activity, goods or services presented on the site. In simple terms, we are talking about the totality of the words that the site will be found by the user in a search engine.

With the help of the Yandex service https://wordstat.yandex.ru/, key phrases were selected, which will be used to advance the site. The following searches are popular for this topic: sushi Krasnoyarsk, sushi delivery, sushi Krasnoyarsk delivery, order sushi, as well as queries containing the names of certain companies. The following key phrases were selected for the site: sushi boom, sushi Krasnoyarsk, sushi Krasnoyarsk delivery, sushi boom, sushi boom Krasnoyarsk, sushi boom, sushi boom Krasnoyarsk, order sushi Krasnoyarsk. Based on the given semantics, the site content was filled: headers, names and descriptions of pages for search engines were compiled based on the received list of keywords (Figure 5).

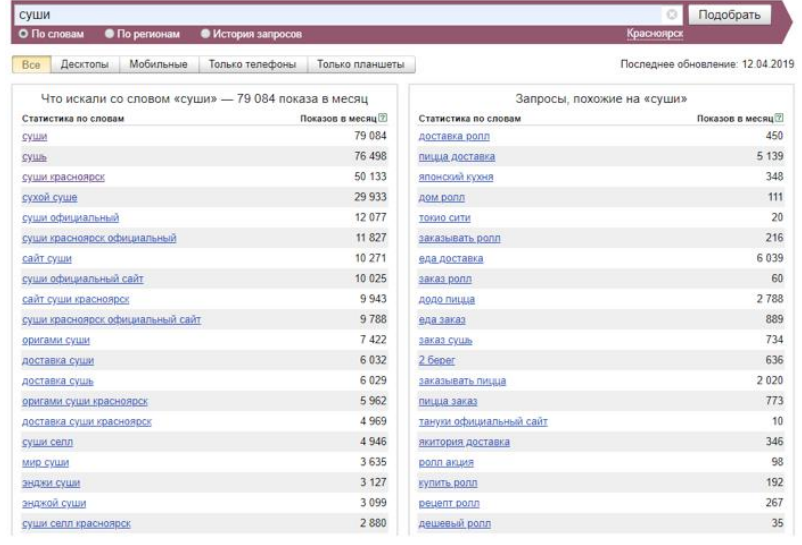

Fig. 5. Site content (web-site language is Russian)

Introduced micro-marking schema.org. Schema.org is a semantic data mark-up dictionary maintained by all major search engines. Its goal is to help web developers create high-quality metadata that will allow search robots to better understand the content of the page and thereby improve the results of the issue. Metadata using the schemas described on schema.org is directly analysed by search robots (Figure 6).

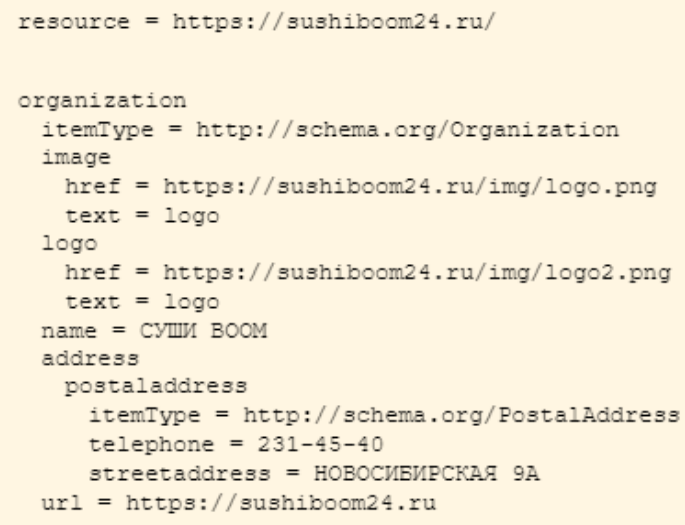

Fig. 6. Schema.org schema

\section{Tags making}

The $<$ Title $>$ tags are an HTML element that is located in the $<$ head $>$ section of the HTML code of the web page. The title tags are important because they allow readers to know what information will be present when the user clicks on the page. They are important to search engines because they also serve to determine the relevance of a web page as a result of a search query. When compiling the headers, it was taken into account that the header tags should be between 50 and 60 characters long. Otherwise, he may be circumcised in the issue. The most relevant keywords were entered into the title, at the same time corresponding to the topics of the pages. Each page has been assigned a title consisting of three parts. First we put the name of the company «Sushi Boom». Further, the word «Delivery» was chosen for the Main page, and for the Menu and Contacts, respectively, the «Menu» and «Contacts». These words allow you to optimize the 
page for their respective requests. The use of the word «Home» in the title is not recommended. The third part of the title is the same for all pages - «Krasnoyarsk». This word we can say set the regional identity of the site.

The $<$ Description $>$ meta tag is a short paragraph of text in the $<$ head $>$ section of the HTML page. This is an important element of the page that helps search engines determine the theme of the page and allows users to understand whether the page matches their query. Although the meta description is not a ranking factor, it does determine whether the user will click on the page, which affects the click-through rate. In addition, an accurate description that matches the content will significantly reduce the bounce rate. This is because the Description tag is included in the snippet.

A snippet is a separate search result in a search result set, which usually consists of a title, a URL, and a page description. The contents of the snippet correspond to parts of the search query, and the keyword will be highlighted in bold in the description of the snippet. This makes the link to the site more attractive to users (Figure 7).

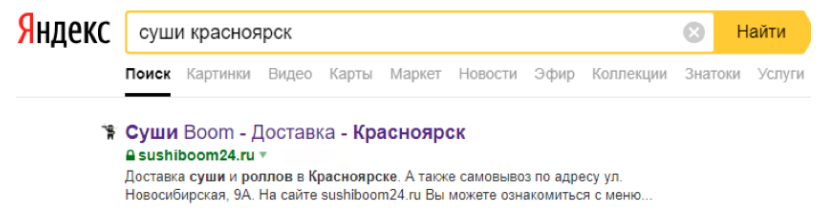

Fig. 7. The search result with snippet (web-site language is Russian)

\section{The experimental results}

On the Yandex.Web service, the master checked the absence of technical errors, as well as the main indicators of the site.

The site has a low SQI of 10. The site's quality index is an indicator of how useful your site is to users from a Yandex point of view. When calculating the quality index, the site's audience size, user satisfaction, and Yandex's and other criteria. The calculation uses data from Yandex services.

Without SEO optimization, the site could only be found by direct request «sushiboom24.ru». After the first changes, he appeared on page 17 for the request «Sushi Krasnoyarsk» in the Yandex search system, specifying the location of the search as Krasnoyarsk city. At this stage of the promotion, the site goes on page 11 . Obviously, most users will not reach this page, but the number of results for this query is 5 million. Also, do not forget about advertisements that occupy a large number of issuing positions. The request «Krasnoyarsk Sushi» is very popular and in demand, therefore, it was first necessary to promote the site by direct target request by the name of the organization. At the request of the «sushi boom» site takes the second place in the issue after the advertisement (Figure 8).

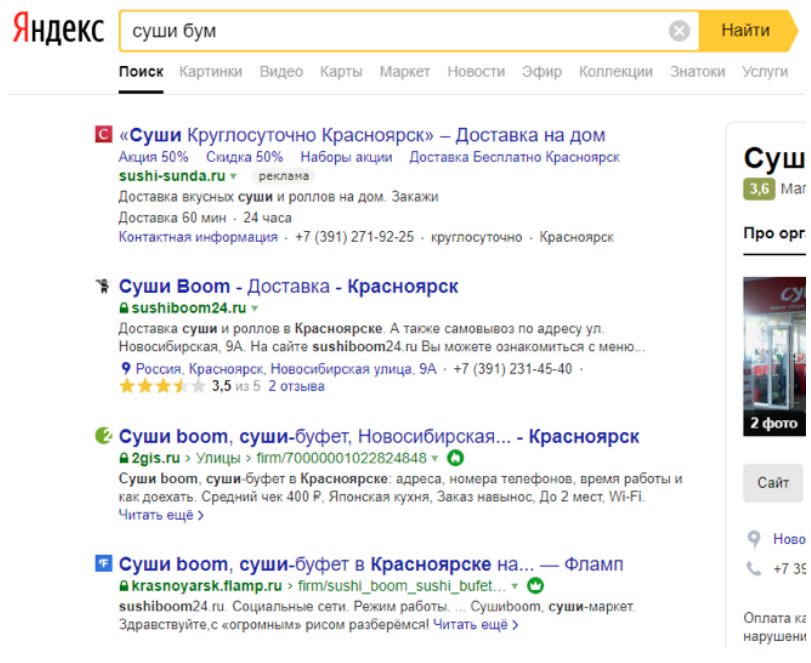

Fig. 8. The search result of web-site made (web-site language is Russian)

\section{Conclusions}

SEO promotion of a site directory in a competitive niche is a difficult task. A large amount of search results and advertisements do not allow bringing the site to higher positions. Large online stores with a basket and the possibility of online ordering move the site directory to the last places in the ranking.

But still, with the help of the correct basic SEO-site settings without using paid advertising, we were able to raise the position of the site by a direct request for the name of the organization with the exact location.

\section{References}

1. Scientific and electronic library An Analysis of Factors Used in Search Engine Ranking Retrieved from: http://airweb.cse.lehigh.edu/2005/bifet.pdf/

2. J.P. Dotson, R.R. Fan, E.M. Feit, J.D. Oldham, Y.H. Yeh, J. of Interactive Marketing, 37 (2017)

3. M.P. Evans, Int. Res. 17, 1 (2007)

4. L.A. Granka, T. Joachims, G. Gay, Twenty-Seventh Ann. Int. ACM SIGIR Conf. on Res. and Dev. in Inf. Retr. (2004)

5. B.J. Jansen, M. Resnick, J. of the Am. Soc. for Inf. S. and Tech. 57, 14 (2006)

6. D. Lewandowski, F. Kerkmann, S. Rümmele, S. Sünkler, J. of the Assoc. for Inf. S. and Tech. 69, 3 (2018)

7. L. Moreno, P. Martinez, Online Inf. Rev. 37, 4 (2013)

8. R. Sen, Int. J. of Electronic Commerce 10, 1 (2005)

9. A. Gandour, A Regolini, Hi Tech News 28, 6 (2011)

10. J. Zhang, A. Dimitroff, Inf. Proc. and Man. 41, 3 (2005)

11. V.V. Kukartsev, E.A. Chzhan, V.S. Tynchenko, O.A. Antamoshkin, A.A. Stupina, J. of Sib. Fed. Uni. - Hum. and Soc. S. 11, 5 (2018) 
12. S. Lee, R.J. Koubek, Comp. in Industry 4, 61 (2010)

13. G. Matosevic, Art. Int. and Alg. in Int. Sys. 8, 12 (2019)

14. C.J. Luh, S.A. Yang, T.L.D. Huang, Online Inf. Rev. 2, 40 (2016)

15. R. Berman, Z. Katona, Marketing S. 4, 32 (2013)

16. N. Hariri, Online Inf. Rev. 4, 35 (2011)

17. J.B. Killoran, IEEE Trans. on Prof. Comm. 1, 56 (2013)
18. S. Sagot, A.J. Fougeres, E. Ostrosi, P. Lacom, Int. Conf. on Inf. Soc. (2014)

19. S. Duk, D. Bjelobrk, M. Carapina, 36th Int. Convention on Inf. and Comm. Tech., Electr. and Microelectr. (2013)

20. J.B. Killoran, IEEE Trans. on Prof. Comm. 3, 52 (2009)

21. S. Gupta, N. Rakesh, A. Thakral, D.K. Chaudhary Fourth Int. Conf. on Parallel, Distr. and Grid Comp. (2016) 